\title{
Anaesthetic implications during off-pump coronary artery bypass surgery
}

\author{
Manjula Sarkar ${ }^{1, *}$, Vishal Prabhu ${ }^{2}$ \\ ${ }^{1}$ Professor and Head, ${ }^{2}$ Senior Resident, Dept. of Cardiac Anaesthesia, Seth G. S. Medical College \& KEM Hospital, Mumbai, \\ Maharashtra, India
}

*Corresponding Author:

Email: drmanjusarkar@gmail.com

Received: $5^{\text {th }}$ July, 2018

Accepted: $29^{\text {th }}$ August, 2018

\begin{abstract}
Off-pump coronary artery bypass has emerged as a popular method of coronary revascularization to mitigate the harmful effects of cardiopulmonary bypass. It requires surgical and anaesthetic expertise for good results. Newer minimally invasive approaches to off pump coronary bypass provide attractive alternatives to conventional off pump surgery, especially in achieving better cosmetic results and early extubation. They require good perioperative pain management, with epidural and paravertebral block being good choices. Emergency conversion of off-pump bypass to on-pump bypass is associated with poor outcomes, so preventive measures to avoid it should be employed.
\end{abstract}

Keywords: Off-pump coronary artery bypass, Anaesthetic implications.

\section{Introduction}

Coronary artery bypass grafting (CABG) was first performed on a beating heart before the development of cardiopulmonary bypass (CPB). With the development and increasing safety of $\mathrm{CPB}$, CABG began to be performed using CPB. Since the 1990s, off-pump coronary artery bypass (OPCAB) has regained popularity, the rationale being to avoid the deleterious effects of CPB. OPCAB can be done via either a sternotomy or minimally invasive approach. Minimally invasive OPCAB requires one-lung ventilation and the access for multiple vessel revascularization may be difficult. Conventional OPCAB avoids these limitations, while retaining rapid access to $\mathrm{CPB}$ in emergencies.

Although many studies have been conducted comparing OPCAB with on-pump CABG, there is no definitive data to suggest the benefit of one procedure over another, with regard to long-term graft patency rates, need for repeat revascularization, stroke and mortality. ${ }^{1-3}$ This is mainly because of the surgeondependent outcome in OPCAB. The patients that are expected to benefit the most from OPCAB include patients with poor left ventricular (LV) function, renal, or liver dysfunction, and those with calcified/atheromatous aortas in whom neurological outcome might be better as aortic cannulation and clamping is minimized. OPCAB demands a high level of vigilance on the part of the anaesthesiologist. This article aims to provide an overview of anaesthetic implications during OPCAB.

\section{Advantages of $\mathrm{OPCAB}^{1-3}$}

1. Reduced systemic inflammatory response, renal dysfunction, short-term neurocognitive dysfunction

2. Reduced need for transfusion, reoperation for bleeding

3. Reduced incidence of atrial fibrillation
4. Reduced need for inotropes

5. Shorter period of postoperative ventilation

6. Reduced intensive care unit (ICU) and hospital stay, costs

\section{Limitations of OPCAB}

Contraindications to OPCAB are intracardiac thrombus, malignant arrhythmias, deep intramyocardial bridging vessels, and concomitant open-heart procedures.

\section{Surgery and Anaesthesia Technique}

Preoperative assessment should include a review of the angiogram and a discussion with the surgeon regarding the surgical plan including the order of grafting. The CPB machine, perfusionist and facilities for defibrillation, cardiac pacing and IABP should be available. Preoperative prophylactic intra-aortic balloon pump (IABP) insertion may be considered in high-risk patients with severe LV dysfunction and left main disease as it has been shown to decrease postoperative low cardiac output syndrome and risk of death. ${ }^{4}$

Routine monitoring for $\mathrm{OPCAB}$ should include 5lead electrocardiogram (ECG) with automated ST segment analysis, pulse oximetry, invasive blood pressure, central venous pressure, end-tidal carbondioxide, urine output, transesophageal echocardiography (TEE) and temperature. If the cardiac surgical plan includes radial artery harvesting, alternative sites of pressure monitoring should be obtained. Additional monitoring like depth of anaesthesia and neuromuscular monitoring can help in fast-tracking of extubation. Normothermia is essential, which is achieved with a warm operating room, warming mattress and fluid warmer.

TEE is done to confirm preoperative cardiac diagnoses, monitor preload, ventricular function, valvular regurgitation and regional wall motion 
abnormalities (RWMA). During heart displacement, TEE views may be lost. The use of stabilizers can produce artifacts of RWMAs. After revascularization, TEE may show new RWMAs which may be associated with reperfusion injury and myocardial stunning. Persistent and severe new RWMAs are correlated with postoperative cardiac complications. ${ }^{5}$

Pulmonary artery catheter can be inserted in selected patients with severe LV dysfunction (ejection fraction $<30 \%$ ), pulmonary hypertension and right-sided heart failure to provide information regarding pulmonary artery pressure (PAP), cardiac output (CO), and mixed venous oxygen saturation. Large $\mathrm{v}$ waves on the pulmonary capillary wedge pressure (PCWP) tracing may indicate myocardial ischaemia or severe mitral regurgitation.

ACT is maintained between 250-300 seconds, with an initial heparin dose of $2 \mathrm{mg} / \mathrm{kg}$. During proximal coronary anastomosis, arterial pressure should be reduced to $90 \mathrm{~mm} \mathrm{Hg}$ to reduce the risk of dissection on application of aortic side-biting clamps. The left internal mammary to left anterior descending artery (LAD) anastomosis is done first as the LAD supplies a large myocardial territory and requires minimal displacement of the heart. Ventilation with low tidal volumes is required during left internal mammary artery (LIMA) harvesting and distal anastomoses to improve exposure. This may cause atelectasis in the postoperative period. Excessive tidal volumes can cause graft compression, especially when bilateral mammary arteries are used.

Access to the coronary arteries on the posterior and inferior surfaces requires the heart to be elevated by using slings, pericardial sutures, surgical packs or a suction device like the Starfish Heart Positioner. Concerns about haemodynamic status when the heart is first positioned should be communicated to the surgeon because once the coronary artery is opened, the surgical options become limited until completion of the anastomosis. Access also requires the Trendelenberg position, which beneficially increases venous return. Tissue stabilizers like the Octopus device (Figure 1) use suction to fix the target coronary artery to facilitate distal anastomosis. Blood flow can be controlled using a suture applied around the coronary artery proximal to the graft site. A blower using humidified carbon dioxide is used to prevent blood obscuring the arteriotomy site. Once opened, a shunt can be inserted into the artery, reducing blood in the surgical field, and allowing perfusion of the distal myocardium.

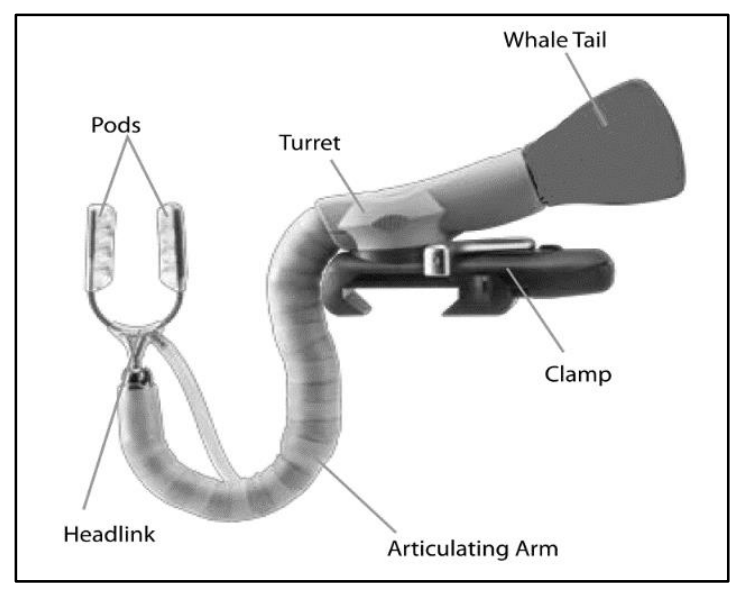

Fig. 1: Medtronic Octopus tissue stabilizer

The ECG may be distorted during cardiac displacement; the axis, amplitude and ST segment changes may be reduced. Causes of hemodynamic compromise are impairment of venous return, impaired ventricular filling, valvular regurgitation, compression of thin-walled right ventricle against the interventricular septum and ischaemia due to occlusion of the target artery. The amount of haemodynamic compromise usually is greatest during rotation of the heart for grafting the circumflex artery and its branches. The surgeon may open the right pleura to reduce compression. Resistant bradycardia especially during grafting of the proximal right coronary artery (RCA) can indicate sinoatrial node ischaemia, and epicardial pacing is urgently indicated. Hypotension could be due to hypovolaemia caused by occult blood loss into the pleural spaces. Severe mitral regurgitation, which can also be due to myocardial ischaemia can cause a fall in oxygen saturation. Early repositioning of the heart is required. Cardiac displacement increases the risk of arrhythmias. Electrolytes, particularly potassium and magnesium should be kept normal in order to avoid reperfusion arrhythmias. Optimizing preload, and the use of vasopressors, inotropes will improve cardiac output. Choice of inotropes/vasopressors include noradrenaline, adrenaline, dopamine, dobutamine, milrinone and levosimendan, which should be guided by patient hemodynamics and institutional protocol. At our institution, we start an infusion of noadrenaline and levosimendan post-intubation if deemed necessary. In selected patient with left main disease and/or severe LV dysfunction, we have started levosimendan on the night prior to surgery with good results. Levosimendan ${ }^{6}$ offers the advantage of increasing contractility and decreasing afterload without significantly increasing myocardial oxygen consumption. CVP and PCWP may not accurately reflect LV filling pressures, especially in patients with valvular pathology and diastolic dysfunction. TEE can help in guiding fluid management during OPCAB surgery. Minimally invasive cardiac output monitors utilizing pulse contour analysis have shown good correlation with intermittent 
thermodilution ${ }^{7}$ and transesophageal echocardiography ${ }^{8}$ measured cardiac output. They can be used to guide fluid management and optimize preload during OPCAB. Post anastomosis, protamine may be partially or fully reversed.

\section{Minimally invasive OPCAB}

Minimally invasive OPCAB involves a thoracotomy, partial sternotomy or subxiphoid incision in contrast to a full sternotomy. It can be performed via direct, endoscopic or robotic access. Avoidance of full sternotomy which is required in conventional OPCAB is beneficial in patients with a prior sternotomy or bypass grafts. The disadvantages of minimally invasive approaches are less adequate exposure to achieve multivessel revascularization and increased duration of surgery. The advantages include potential for improved cosmesis and wound healing along with reduced morbidity, pain, cost, transfusion and length of stay in the ICU. ${ }^{12}$ Patient selection is crucial in ensuring success of these techniques due to limited access to surgical site, requirement of one-lung ventilation, potential for incomplete revascularization and postoperative pain management. Provision for external defibrillation is a must in minimally invasive cardiac surgery. Endoscopic CABG will require insufflation of carbon-dioxide along with one-lung ventilation. Continuous monitoring of insufflation pressure, airway pressure and central venous pressures is required. One-lung ventilation has potential to cause hypoxia and may require use of permissive hypercarbia. This may necessitate use of inotropes and cause heart irritability, both of which are undesirable in performing revascularization on a beating heart.

\section{Pain Management}

Adequate control of pain is crucial for fast-tracking extubation of cardiac surgery patients. Thoracotomy is considered to be more painful than median sternotomy. Regional anaesthetic techniques are known to reduce opioid consumption, reduce stress response, improve coronary blood flow and pulmonary function, and facilitate early ambulation. ${ }^{7-12}$ Commonly used regional techniques include epidural block, paravertebral block, intrathecal opioids, intercostal nerve block and intrapleurl block. Catheters for regional analgesia should be inserted the day before surgery or at least an hour prior to heparinization. Surgery should be delayed for 24 hours in the event of a traumatic tap. Standard guidelines should be followed to ensure safe removal of these catheters post surgery if the patient is receiving heparin.

Complications of epidural blockade are hypotension, intravascular injection and epidural hematoma. Thoracic paravertebral block is comparable to thoracic epidural anaesthesia in procedures involving unilateral thoracotomy incision. ${ }^{13}$ It can decrease the risk of epidural hematoma while avoiding the hemodynamic effects of the sympathectomy seen with neuraxial blockade. ${ }^{14-16}$ Complications of paravertebral block are vascular puncture and pleural puncture with pneumothorax. Despite proven benefits and the low risk of epidural hematoma, these techniques are yet to gain widespread acceptance. NSAIDs and paracetamol are important adjuncts in multimodal analgesia.

\section{Complications and Conversion to On-pump}

Complications of OPCAB are severe hypotension unresponsive to inotropes, malignant arrhythmias, new regional wall motion abnormalities, or complete cardiovascular collapse. They require emergency conversion to on-pump CABG. Conversion to CPB has been shown to increase morbidity and mortality with operative mortality ranging from 8.5 to $18 \%$. Congestive heart failure, redo surgery, low ejection fraction, recent myocardial infarction, mitral regurgitation, and chronic obstructive pulmonary disease have been reported as independent predictors of emergency conversion to CPB.

\section{Summary}

Cardiopulmonary bypass is a safe technology that has led to innovations in cardiac surgery with better outcomes. Knowledge of complications associated with it is important to avoid catastrophes. Good communication between the entire surgical team is vital. OPCAB, with good surgical and anaesthetic experience can provide similar results to on-pump CABG, along with certain specific advantages.

\section{Acknowledgements}

We are thankful to Dr. Avinash Supe, Dean, Seth G.S. Medical College \& KEM hospital for his encouragement to our academic activities.

\section{Financial Support and Sponsorship: Nil.}

\section{Conflicts of Interest: None.}

\section{References}

1. Cheng DC, Bainbridge D, Martin JE. Does off-pump coronary artery bypass reduce mortality, morbidity, and resource utilization when compared with conventional coronary artery bypass? a meta-analysis of randomized trials. Anaesthesiology. 2005;102:188.

2. Raja SG, Dreyfus GD. Off-pump coronary artery bypass surgery: to do or not to do? Current best available evidence. J Cardiothorac Vasc Anaesth. 2004;18:486.

3. Sellke FW, Di Maio JM, Caplan LR. Comparing onpump and off-pump coronary artery bypass grafting: numerous studies but few conclusions: A scientific statement from the American Heart Association Council on Cardiovascular Surgery and Anaesthesia in collaboration with the Interdisciplinary Working Group on Quality of Care and Outcomes Research. Circulation. 2005;111:2858.

4. Yang F, Wang J, Hou D, Xing J, Liu F, Xing ZC. Preoperative intra-aortic balloon pump improves the clinical outcomes of off-pump coronary artery bypass grafting in left ventricular dysfunction patients. Sci Rep. 2016;6:27645. 
5. Gurbuz AT, Hecht ML, Arslan AH. Intraoperative transesophagial echocardiography modifies strategy in off-pump coronary artery bypass grafting. Ann Thorac Surg. 2007;83:1035-40.

6. Zenati M, Domit TM, Saul M, et al. Resource utilization for minimally invasive direct and standard coronary artery bypass grafting. Ann Thorac Surg. 1997;63:S84S87.

7. Blomberg S, Curelaru I, Emanuelsson H. Thoracic epidural anaesthesia in patients with unstable angina pectoris. Eur Heart J. 1989;10:437.

8. Gramling-Babb P, Miller MJ, Reeves ST, et al. Treatment of medically and surgically refractory angina pectoris with high thoracic epidural analgesia: initial clinical experience. Am Heart J. 1997;133:648.

9. Olausson K, Magnusdottir H, Lurje L. Anti-ischemic and anti-anginal effects of thoracic epidural anaesthesia versus those of conventional medical therapy in the treatment of severe refractory unstable angina pectoris. Circulation. 1997;96:2178.

10. Overdyk FJ, Gramling-Babb PM, Handy JR Jr. Thoracic epidural anaesthesia as the last option for treating angina in a patient before coronary artery bypass surgery. Anaesth Analg. 1997;84:213.

11. Gramling-Babb PM, Zile MR, Reeves ST. Preliminary report on high thoracic epidural analgesia: Relationship between its therapeutic effects and myocardial blood flow as assessed by stress thallium distribution. J Cardiothorac Vasc Anaesth. 2000;14:657.

12. Onan IS, Onan B, Korkmaz AA, et al. Effects of thoracic epidural anaesthesia on flow and endothelium of internal thoracic artery in coronary artery bypass graft surgery. $J$ Cardiothorac Vasc Anaesth. 2011;25:1063.
13. Davies RG, Myles PS, Graham JM. A comparison of the analgesic efficacy and side-effects of paravertebral vs epidural blockade for thoracotomy - A systematic review and meta-analysis of randomized trials. Br J Anaesth. 2006;96:418-26.

14. Mehta Y, Arora D, Sharma KK, Mishra Y, Wasir H, Trehan N. Comparison of continuous thoracic epidural and paravertebral block for postoperative analgesia after robotic-assisted coronary artery bypass surgery. Ann Card Anaesth. 2008;11:91-6.

15. Dhole S, Mehta Y, Saxena H, Juneja R, Trehan N. Comparison of continuous thoracic epidural and paravertebral blocks for postoperative analgesia after minimally invasive direct coronary artery bypass surgery. J Cardiothorac Vasc Anesth. 2001;15:288-92.

16. Ganapathy S, Murkin JM, Boyd DW, Dobkowski W, Morgan J. Continuous percutaneous paravertebral block for minimally invasive cardiac surgery. J Cardiothorac Vasc Anesth. 1999;13:594-6.

How to cite this article: Sarkar M, Prabhu V. Anaesthetic implications during off-pump coronary artery bypass surgery. Indian J Clin Anaesth. 2018;5(3):311-314. 